\title{
Determining whether previous SWL for ureteric stones influences the results of ureteroscopy as the second-line treatment: A clinical study
}

\author{
CĂTĂLIN PRICOP ${ }^{1,2}$, MARIUS IVANUTA $^{2}$, GEORGE DANIEL RADAVOI ${ }^{3,4}$, CRISTIAN-VALENTIN TOMA $^{3,4}$, \\ ALIN CUMPANAŞ $S^{5,6}$, VIOREL JINGA ${ }^{3,4}$, NICOLAE BACALBAŞA ${ }^{3,7}$ and DRAGOŞ PUIA ${ }^{1,2}$ \\ ${ }^{1}$ Department of Urology, 'Grigore T. Popa' University of Medicine and Pharmacy, 700115 Iași; ${ }^{2}$ Department of Urology, \\ 'C. I. Parhon' Hospital, 700503 Iași; ${ }^{3}$ Department of Urology, 'Carol Davila’ University of Medicine and Pharmacy, \\ 020021 Bucharest; ${ }^{4}$ Department of Urology, 'Prof. Dr. Theodor Burghele' Clinical Hospital, 050653 Bucharest; \\ ${ }^{5}$ Department of Urology, 'Victor Babeş’ University of Medicine and Pharmacy, 300041 Timișoara; ${ }^{6}$ Department of Urology, \\ 'Pius Brînzeu' County Emergency Clinical Hospital Timiş, 300723 Timişoara; ${ }^{7}$ Department of Visceral Surgery, \\ Center of Excellence in Translational Medicine, 'Fundeni’ Clinical Institute, 022328 Bucharest, Romania
}

Received August 19, 2021; Accepted September 20, 2021

DOI: $10.3892 / \mathrm{etm} .2021 .10960$

\begin{abstract}
The purpose of the study was to establish whether shock wave lithotripsy (SWL) is a potential harmful first-line treatment for ureteric stones where ureteroscopy (URS) is necessary as a second-line treatment. Medical records of patients with ureteric stones who underwent either URS as the only therapy applied or SWL followed by URS over two years were retrospectively evaluated. In total, 158 patients were included: 79 patients in Group A (no SWL) and 79 in Group B (prior SWL before URS). There was no difference in major complications, Group A had higher stone-free rates, Group B had higher rates of ureteral edema and similar intraoperative ureteral lesions. In conclusion, the failure of SWL for lumbar or pelvic ureteral lithiasis does not appear to have a negative effect on the rate of intraoperative complications or the success rate of semi-rigid retrograde URS for this category of calculi, with the same safety profile as first-line endourological intervention.
\end{abstract}

\section{Introduction}

Kidney stone disease is a prevalent condition worldwide with a prevalence of 4-20\% in developed countries and epidemiological data show an increasing incidence (1). With the recent technological advances, extracorporeal shock wave lithotripsy

Correspondence to: Dr George Daniel Radavoi, Department of Urology, 'Carol Davila' University of Medicine and Pharmacy, 37 Dionisie Lupu Street, 020021 Bucharest, Romania

E-mail: radadaniel@yahoo.com

Key words: shock wave lithotripsy, semirigid ureteroscopy, ureteral edema, stone-free, ureteral lithiasis
(ESWL) and retrograde ureteroscopy (URS) with or without intracorporeal lithotripsy of the renal stones, have become the most frequently used therapeutic methods for nephrolithiasis. Both methods have advantages and disadvantages. SWL is non-invasive, has both a low complication rate and reduced anesthetics use $(2,3)$. However, according to Pearle (3) and Larcher et al (4), SWL for a stone larger than $1 \mathrm{~cm}$ has a lower success rate in comparison with URS $(4,5)$. By contrast, URS is a more invasive procedure requiring general or regional anesthesia, and frequent hospitalization, and it has a higher infectious complication rate; thus, prophylactic antibiotic therapy is recommended (6).

In the present study, the results of URS with pneumatic lithotripsy, as a first therapeutic method, were compared with those of the URS after a failed SWL for ureteric stones.

\section{Patients and methods}

Patients. The objective of the present study was to determine whether SWL is a limiting and potentially harmful first-line procedure for ureteric stones where URS is necessary as a second-line treatment to render a stone-free status. To achieve this objective, medical records of patients with ureteric stones who underwent either URS as monotherapy or SWL followed by URS (where SWL monotherapy failed to render the stone-free status) were retrospectively evaluated, between January 2017 and December 2019.

The 158 patients were included in two groups ( $\mathrm{n}=79$ each): Group A comprised patients with no SWL before URS, and Group B comprised patients who previously had SWL. Further patient data are provided in Table I. If after more than two SWL sessions the X-ray did not show the disintegration of the stone two weeks from the last procedure, it was considered as treatment failure and the patient underwent URS. SWL was performed with a Chinese KS-884 second-generation, electro-hydraulic spark gap lithotripter, while for the URS a semi-rigid, single-channel ureteroscope (Olympus 
Corporation $^{\circledR}$ ) was used. The intracorporeal lithotripsy was performed with a EMS Swiss Lithoclast 2 device.

The study was approved by the 'C. I. Parhon' Clinical Hospital ethics committee, with the number: 12/07.02.2020. Written informed consent was obtained from all the patients. All data were collected from patients charts from the ' $\mathrm{C}$. I. Parhon’ Hospital, Department of Urology (Iași, Romania).

Methods. Demographic data, clinical, radiological, intraoperative findings, and SWL parameters were collected and recorded. In all cases, urine culture was performed to determine a more precise antibiotic prophylaxis. A standard surgical technique was used for URS. A safety guidewire was used during the procedures. No intramural ureteral balloon dilatation was made. After removing the stone, routine retrograde pyelography was conducted, and at the end of the procedure, all the patients underwent a ureteral catheterization with a JJ stent. Quantification of the intraoperative complications was made using the modified Satava classification.

The present study included only procedures performed by surgeons with more than 5 years of experience. Stone-free rate after URS was evaluated through an abdominal X-ray film 4 weeks after the procedure, before the JJ stent removal.

Statistical analysis. SPSS.16 software (SPSS, Inc.) was used to analyze the statistics. Statistical analysis for quantitative data was performed using the Student's t-test (e.g., BMI, stone size) and ANOVA (e.g., operative time between subgroups). while the Chi-square with Yates correction test was used for qualitative data (e.g., stone free rate, gender distribution). $\mathrm{P}<0.05$ was considered to indicate a statistically significant difference.

\section{Results and Discussion}

A total of 158 patients were included, 79 patients in Group A and 79 in Group B. Demographic data, as well as the clinical features and the calculus parameters, are shown in Table I. There were no significant statistical differences between the two groups regarding age $(\mathrm{P}=0.07)$, sex $(\mathrm{P}=0.79)$ or body mass index $(\mathrm{BMI})(\mathrm{P}=0.5)$.

The aspect of the ureteric mucosa on URS is presented in Table II. One can see that previous SWL led to more significant edema of the ureteric mucosa. There was no difference between groups regarding the mean operative time.

The rate of intraoperative complications, according to Satava classification is summarized in Table III.

When selecting the optimal therapeutic approach for lumbar or pelvic ureteric stones, several factors should be considered including stone size, degree of the secondary obstruction, invasiveness of the technique, and cost effectiveness. Although the European Association of Urology recommends SWL as a therapeutic method for pelvic ureteral calculi that are smaller than $1 \mathrm{~cm}$ and did not pass after medical expulsion therapy, for lumbar calculi larger than $1 \mathrm{~cm}$ the indication remains a subject for controversy, leaving the choice between URS and SWL to be decided by the patient and the urologist $(5,6)$.

The therapeutic approach of nephrolithiasis has markedly changed over the last two decades through great technological progress (7). The availability of minimally invasive procedures such as stenting, endoscopic extraction of calculi, and ESWL are highly efficient procedures that, with the hands of experienced surgeons, have almost made classical surgical interventions for nephrolithiasis a relic of the past (5). Nowadays, SWL is safer and less invasive than retrograde URS, with minimal adverse effects and also the damage of the shockwaves to the tissue is reversible in a matter of days (6).

ESWL has a success rate that can be decreased by the calculus parameters (size, structural composition such as brushite, monohydrate calcium oxalate, and cystine calculi are more resistant to ESWL, and location) and also by the patient's parameters (such as obesity, abdominal circumference, or malformations of the urinary tract) $(7,8)$. According to Picozzi et al with each millimeter over the $8 \mathrm{~mm}$ threshold of the calculus diameter, the probability of the stone-free status of the patient decreases by $5 \%$ regarding pelvic calculi and $8 \%$ regarding lumbar calculi (9).

Few studies have focused on the efficiency and safety of the retrograde URS after an SWL failure. Despite the well-defined advantages of the ESWL, such as the patient's comfort and the satisfactory results, there is a question being raised by many authors, namely if an SWL session with no gratifying results has a negative impact on the results of a possible future URS. This assumption comes from the inferences of recent studies that proved that the shockwaves generated by the lithotripsy device trigger an inflammatory tissue response, releasing prostaglandins, cyclooxygenase-2 (COX-2), and tumor necrosis factor $(\mathrm{TNF} \alpha)$, which lead to tissue damage, edema, and low blood flow, the aftermath being increased fragility of the ureteral mucosa and of the blood vessels, all in all, complicating ureteral surgery, including endoscopic treatment $(10,11)$.

Regarding the stone-free rate of SWL versus URS, the data are still conflicting. Park et al (12) asserted that for lumbar calculi smaller than $10 \mathrm{~mm}$ the success rate after one lithotripsy session is $84.3 \%$ (12). As for larger calculi, such as $20-30 \mathrm{~mm}$, Abe et al (13), on a group of 267 patients, reported a stone-free rate of $65.1 \%$ in patients on which SWL was used as a monotherapy. In a recent study, Ur Rehman et al (14) compared the results of SWL and URS in the treatment of lumbar calculi smaller than $20 \mathrm{~mm}$. The authors obtained a stone-free rate of $64 \%$ after the first session, $77.3 \%$ after the second, and $94.7 \%$ after the third session, as for URS the authors had a stone-free rate of $86.7 \%$ after the first endoscopic intervention (14). Partially contradictory, on a cohort of 657 patients with pelvic urolithiasis (10-15 mm), Dell'Atti and Papa (15) reported stone-free rates of $77.5 \%$ with retrograde URS and $45.4 \%$ with ESWL only. Of course, the compared data have different operators who perform ESWL, probably with different energy and frequency strategies. Another aspect that contributes to the bias of the statements is the ESWL-selected position which varies from center to center, with Soliman et al (16) suggesting that a more favorable efficacy profile and patient satisfactory rate for distal ureteral stones is achieved through the transgluteal or supine approach.

In a group of 93 patients, Holland et al (17) found that if retrograde intrarenal surgery (RIRS) for urolithiasis is conducted after SWL, there are higher morbidity rates than if it were conducted as first-line therapy. Thus, the authors found a stone-free rate of $80 \%$ in patients who had the first-line URS, in comparison to $67 \%$ success rate in patients who received URS after ESWL failure; this phenomenon is explained by the 
Table I. Patient characteristics, surgical features, and intraoperative complications.

\begin{tabular}{|c|c|c|c|}
\hline Characteristics & Group A & Group B & P-value \\
\hline Number of patients & 79 & 79 & \\
\hline Females $(\%)$ & $44.3 \%$ & $39.2 \%$ & 0.79 \\
\hline Males $(\%)$ & $55.7 \%$ & $60.8 \%$ & \\
\hline Mean age (years) $( \pm \mathrm{SD})$ & $46.3( \pm 11.31)$ & $49.5( \pm 9.67)$ & 0.07 \\
\hline $\mathrm{BMI}\left(\mathrm{kg} / \mathrm{m}^{2}\right)( \pm \mathrm{SD})$ & $24.84( \pm 3.32)$ & $24.6( \pm 4.95)$ & 0.50 \\
\hline Size of the stone $(\mathrm{mm})( \pm \mathrm{SD})$ & $11.2( \pm 2.36)$ & $13( \pm 3.43)$ & 0.08 \\
\hline Side (cases) & Right (43) & & \\
\hline Left (36) & Right (38) & & \\
\hline Left (41) & 0.52 & & \\
\hline Location of the calculus (cases) & Lumbar ureter (58) & & \\
\hline Pelvic ureter $(21)$ & Lumbar ureter (46) & & \\
\hline Pelvic ureter $(33)$ & 0.04 & & \\
\hline Mean operative time $(\min )( \pm \mathrm{SD})$ & $65.8( \pm 14)$ & $74.9( \pm 13)$ & $<0.05$ \\
\hline Stone-free rate $(\%)$ & $87.35 \%$ & $84.9 \%$ & 0.64 \\
\hline
\end{tabular}

Group A, no SWL; Group B, prior SWL before URS. SWL, shock wave lithotripsy; URS, ureteroscopy; BMI, body mass index.

Table II. Endoscopic aspect of the ureteric mucosa.

\begin{tabular}{lrrr}
\hline Variable & Group A & Group B & P-value \\
\hline Significant edema & $30.37 \%(\mathrm{n}=24)$ & $67.08 \%(\mathrm{n}=53)$ & 0.00001 \\
Calculus impacted in the mucosa & $40.5 \%(\mathrm{n}=32)$ & $54.4 \%(\mathrm{n}=43)$ & 0.15 \\
Retained fragments & $12.65 \%(\mathrm{n}=10)$ & $15.1 \%(\mathrm{n}=12)$ & 0.81 \\
\hline
\end{tabular}

Group A, no SWL; Group B, prior SWL before URS. SWL, shock wave lithotripsy; URS, ureteroscopy.

Table III. Intraoperative complications, according to the modified Satava classification.

\begin{tabular}{lccc}
\hline Variable & Group A & Group B & P-value \\
\hline Minimal mucosal injury & $20.25 \%(\mathrm{n}=16)$ & $25.31 \%(\mathrm{n}=20)$ & 0.56 \\
False passage & $3.8 \%(\mathrm{n}=3)$ & $5.06 \%(\mathrm{n}=4)$ & 0.69 \\
Proximal migration of the calculus & $5.06 \%(\mathrm{n}=4)$ & $3.8 \%(\mathrm{n}=3)$ & 0.69 \\
Major perforation & - & - & 0.64 \\
Minor perforation & $2.5 \%(\mathrm{n}=2)$ & $3.8 \%(\mathrm{n}=3)$ & 0.77 \\
Hematuria & $7.6 \%(\mathrm{n}=6)$ & $10.1 \%(\mathrm{n}=8)$ & \\
\hline
\end{tabular}

Group A, no SWL; Group B, prior SWL before URS. SWL, shock wave lithotripsy; URS, ureteroscopy.

authors, as shockwaves from the SWL caused the entrenchment of the calculi or calculi fragments into the submucosa (17). From the same point of view, Kilinc et al (18) found that, during URS in patients who previously had SWL, pseudomembranes may be responsible for the less satisfying results of these patients. In the present study, the stone-free rates of the two groups were almost similar (Group A: $87.35 \%$ vs. Group B: $84.9 \%$ ), with very little in favor of the cases in which URS was the first-intention therapy, nevertheless statistically irrelevant. Similar to our results, Philippou et al (19) in a group of 36 patients found perfectly equal stone-free rates $(88.9 \%)$, as much in patients who received the first-intention URS as in patients who previously received ESWL (19). The hypothesis of the negative influence that ESWL may have on the results of URS regarding the stone-free rate was also refuted by Kilinc et al (18), who, on a cohort of 555 patients, found a stone-free rate of $81.25 \%$ on patients who received URS as first-line treatment, while on patients who previously received lithotripsy, the rate was $78.75 \%$ $(\mathrm{P}<0.05)(18)$. Furthermore, recent findings suggested that if the first lithotripsy session fails, the endoscopic management of the 
pathology must be considered, especially when it is proximal urolithiasis and the size of the calculus exceeds the $10-\mathrm{mm}$ threshold (14). Regarding the surgical time, we found that for patients who received URS as a first-line treatment, the mean operative time was $65 \mathrm{~min}$, and in Group B the mean operative time was $12.2 \%$ higher, namely $74.9 \mathrm{~min}$. Although the operative time was decreased, the propensity of higher operative time in patients who received ESWL before URS was also validated by the study conducted by Kilinc et al (18), in which the patients who received URS as a single procedure had a mean operative time of $32 \mathrm{~min}$, while in the other group the mean time was 34 min (18). Ur Rehman et al (14) identified a mean operative duration close to results of the present study, i.e., $85 \mathrm{~min}$ for lumbar urolithiasis. Previous findings showed that, the mean operative time on patients treated via URS as first-line therapy was 59 min, while in the second-line URS this was 64 min (19). Another aspect that contributes to the controversy of this subject is the timing aspect as the optimal moment to perform URS after failed ESWL remains unclear.

The longer operative time on patients who received ESWL before the URS can possibly be explained either by the entrenchment of the calculus into the ureteral mucosa or the edema of the ureteric mucosa. Although the common definition of the calculus entrenchment into the ureteric mucosa suggests that the guidewire or the catheter cannot pass over the obstacle on the first attempt, the entrenchment can also be defined as the calculus immobility for more than two months (20).

This could explain the results of the present study, where we found ureteral mucosa edema in patients who received SWL before URS in $67 \%$ of cases; meanwhile, only $30 \%$ of the patients who received the first-intention URS had ureteral mucosa edema. Chaussy and Fuchs (21) described the entrenchment into the mucosa as a common phenomenon after ESWL was closely related to the calculus resistance to the shockwaves. As there is no space for expansion for an impacted ureteric stone, the result of the extracorporeal lithotripsy is not always successful; thus, the futility of the second ESWL in these cases (21).

It is possible that the shockwaves may have caused the entrenchment of the calculus into the mucosa since the entrenchment was observed in $54.4 \%$ of patients from Group B, while in patients from Group A the entrenchment was observed in $40.5 \%$ of cases. It should also be considered that the surgical duration is a good reference point for the surgeon's experience and the calculus' complexity, but it can also be affected by the calculus' location, size, and ureteral distension (22). In the present study, the factors related to the calculus' size and location were negligible, as such there were no significant differences between the two batches, statistically speaking. Thus, these aspects, namely the edema and the calculus entrenchment, but also the surgeon's experience, can be involved in the lengthening of the operative time in the categories of patients examined in the present study.

Overall, URS has minor and self-limiting complications, and only a handful of major complications that require surgical intervention. URS is an important acquirement in a urologist's therapeutic arsenal, but, as is the case with any other surgical intervention, it is not without incidents and complications that can occur at any time during the maneuver, most often resulting in various degrees of damage to the ureter (23).
In the literature, these complications are reported at a rate of $3.5-30 \%(21,23)$. In the present study, the quantification of the intraoperative complications identified was achieved using the Satava system adjusted for URS (24). The Satava classification is a quick, concise and easy system, used to describe the severity of the intraoperative complications found in URS that, at the same time allows facilitation of the comparison between the surgical results of different medical centers.

In the present study, we identified minor ureteral lesions that were considered a part of the Satava 1 category which had no negative influence on the intervention. If the mucosal injury was described on $20.2 \%$ of the patients that received first-intention URS, on the patients that previously received extracorporeal lithotripsy the percentage was slightly higher, $(25.3 \%)$. From this frame of reference, the results from the present study are conflicting with those reported by other authors, meaning it has a higher percentage. For instance, Kilinc et al (18) reported a percentage of only $0.9 \%$ of patients who received first-intention URS and $1.62 \%$ who received lithotripsy before URS. The percentage of superficial mucosal injuries found in the present study was higher than the one reported on a cohort of 2,436 patients who received URS, which shows a percentage of $1.5 \%$ mucosal lesions (mucosal abrasions) (25). Other authors reported different percentages of this complication. Ibrahim (26) in a group of 148 patients who received URS, reported superficial ureteral lesions on 9.5\% of patients. Almusafer and Al-Tawri (27) found that 11 out of 18 patients (57.9\%) who received SWL before URS, suffered ureteral mucosa abrasions, while Fuganti et al (28), on a cohort of 1,235 ureteroscopies, reported an almost similar percentage of minor ureteral lesions, namely $61.8 \%$. A study conducted by Ogreden et al (29) on early URS for distal ureteral stones highlighted the efficiency of this procedure in experienced hands with rather low complication rates, similar to our study. Therefore, significant discrepancies regarding the reports on this type of complication should be investigated. According to the generally accepted definition, 'intraoperative complication' (30) means any deviation from the ideal itinerary of a surgical intervention that can interfere with postoperative evolution, therefore this discrepancy may reside in the under-reporting of the endoscopic aspect of the ureteral mucosa as this complication does not modify the results of the surgical intervention.

In the present study, we identified 6 cases of proximal migration of the calculi, 3 in each group (3.8\%), each case being a calculus located in the lumbar ureter. No stone antiretropulsion devices were available during our procedures which may explain the slightly elevated percentage in comparison to other reports of this intraoperative incident. From this point of view, Irer described equal calculus migration percentages in patients who received first-intention URS as well as in patients in which URS followed extracorporeal lithotripsy (2.4\%), although higher percentages of calculi migration to the calyx were reported (10). Findings of a previous study showed percentages of $14.4 \%$ in the case of impacted calculi and $7.9 \%$ in the case of non-impacted calculi, thereby affirming that the degree of the calculus entrenchment is an important predictive factor regarding the risk of proximal migration (27).

Another complication found intraoperatively is the iatrogenic false passage. According to the definition, a false passage 
represents the crossing of the ureteral mucosa and submucosa, with one of the instruments used in URS that produces a tunnel, but without perforating the ureter. Although most of the time this complication is classified as minor, there are specific situations in which the aggression on the ureter's vascularization can produce ureteral necrosis (20). In the present study, similar percentages of this complication was found in both groups $(3.8 \%)$. In a larger cohort, Kilinc et al (18) reported a percentage of $0.36 \%$ in patients who received URS after lithotripsy and $0 \%$ in patients who received URS per primam, while Almusafer and Jawad Al-Tawri yielded a percentage similar to ours, i.e., 2.25\% (27). Geavlete et al (25) estimated that false passage occurs in $1 \%$ of the first-intention ureteroscopies, whether it be diagnostic or therapeutic.

Within the present study groups, we did not find the presence of major ureteral perforations, but we identified 2 major, or even 3 minor perforations in Group B ( $\mathrm{P}>0.05)$. In the present study, the 5 perforations corresponded to grade 1 on the scale of ureteral lesions proposed by Traxer and Thomas (31). Fuganti et al (28) reported that ureteral perforations in patients of that study accounted for $14 \%$ of all intraoperative complications while finding an occurrence rate of $0.65 \%$ in 1,235 ureteroscopies performed, a percentage which is identical to that found by Geavlete et al (25), while Tepeler et al (32) identified a much lower percentage, i.e., $0.16 \%$ of urethral perforations in a group of 1,208 patients. Kilinc et al (18) reported a similar incidence to other authors, that of $0.18 \%$. A higher percentage, similar to the one reported by us, was also found by Ogreden et al (28) who identified $4.6 \%$ ureteral perforations on a group of 811 patients, while at the opposite end is Philippou et al (19) who reported 0\% ureteral perforations. In addition, in a comparative study, Almusafer and Jawad Al-Tawri (27) reported an 8.6 and 1.5\% incidence of minor and major ureteral violence, respectively. In our groups of patients, these minor perforations did not require nephrostomy insertion or open surgery, the solution chosen by the placement of a JJ stent.

Hematuria associated with URS is often minor and self-limiting, usually as a consequence of the ureteroscope advancing through the ureteral orifice, of the abrasion caused by the guidewire or of the mucosal injury during lithotripsy, but also by manipulating fragments during extraction. Generally, hematuria during URS does not change its results until the intervention is stopped due to poor visibility caused by bleeding. In the present study, we found a 7.6 and $10.1 \%$ incidence of hematuria, but in none of the cases was there a need to stop the intervention as the hematuria was insignificant. In addition, Kilinc et al (18) found in their group of patients an incidence of $0.72 \%$, while Geavlete et al (25) reported a percentage of $2.04 \%$ and Almusafer and Jawad Al-Tawri (30) found similar percentages of 1.8\%. By contrast, Nikoobakht et al (7) found a $46.2 \%$ incidence of hematuria after URS. Thus, the reporting of hematuria as a complication of retrograde URS, whether performed by the first intention or after extracorporeal lithotripsy, varies widely. The explanation of this phenomenon could lie in the subjectivism to which the appreciation of the intensity of hematuria is reported. In the present group of patients, we did not have complications such as ureteral avulsion, ureteral invagination, or extra ureteral migration of the calculus.
Finally, radiation exposure is an important aspect that should be considered when selecting the appropriate therapeutic strategy because both SWL and USR may require the use of fluoroscopy. Each patient may receive per stone treatment a mean dose of $5.3 \mathrm{mSv}$, with higher doses in those with renal stones and those who required CT scans and other interventions (32). Previous data showed the air kerma-area product $\left(\mathrm{P}_{\mathrm{KA}}\right.$; all values in $\left.\mathrm{cGy} / \mathrm{cm}^{2}\right)$, for the patients subjected to ESWL for lumbar ureteral lithiasis the mean of $\mathrm{P}_{\mathrm{KA}}\left(\mathrm{cGy} / \mathrm{cm}^{2}\right)$ was 509 ( $\mathrm{SD}=180)$, while for those treated for pelvic ureteral lithiasis the mean of $\mathrm{P}_{\mathrm{KA}}$ was 342 ( $\left.\mathrm{SD}=201\right)$ (33). In the URS group for lumbar ureteral lithiasis, the mean of $\mathrm{P}_{\mathrm{KA}}\left(\mathrm{cGy} / \mathrm{cm}^{2}\right)$ was 892 ( $\mathrm{SD}=436)$, while for patients with pelvic ureteral lithiasis, the mean of $\mathrm{P}_{\mathrm{KA}}$ was $601(\mathrm{SD}=429)$. The risk factors of higher radiation doses were obesity, exposure time, and localization of the stones. With the technological advances in the treatment of kidney stones, radiation exposure is decreased. Abid et al (34) performed ESWL with fluoroscopy combined with ultrasound localization using an 'outline' Automatic Ultrasound Positioning Support (AUPS) and a 'free-line' Visio-Track (VT) (EDAP-TMS) hand-held three-dimensional ultrasound stone locking system in 154 patients. Authors of that study concluded that VT significantly reduced fluoroscopy use during SWL and the duration and dose of patient exposure to ionizing radiation. Additionally, stone treatment efficacy was significantly greater with VT mainly because of better real-time monitoring of the stone (34). Thus, studies should be performed to assess the level of radiation exposure received by practicing physicians and nurses in the two types of groups to assess if the prolonged operative time suggested by URS followed by ESWL leads to radiation surcharge.

Another challenging aspect involves the long-term complications regarding ureteral strictures after ureteral instrumentation and the best practice in correcting them (35). Findings of a study conducted between 2013 and 2018 in an experienced center on 95 patients who developed ureteral strictures after per primam URS showed that reconstruction performs more favorably than endourological procedures. There are no clear data regarding the stricture rate after URS following ESWL and the best-correcting procedure indicated; consequently, further studies are necessary (36).

In conclusion, the failure of SWL for lumbar or pelvic ureteral lithiasis does not appear to have a negative effect on the rate of intraoperative complications or the success of semi-rigid retrograde URS for this category of calculi, with the same safety profile as when endourological intervention is carried out as first-line therapy. Defining minor complications after URS is not always easy, as the literature shows. Calculi whose composition determines an increased hardness are difficult to fragment both by extracorporeal lithotripsy and by contact lithotripsy, because in the case of URS there are also large fragments, often with fractured edges which are difficult to remove, not only small fragments in the form of dust. When compared to first-intention URS, the operative time of endoscopic intervention performed after SWL failure is longer in our statistics; the explanation for this phenomenon being given by more pronounced edema, which causes a poorer visualization of the stone during URS. Despite the risk of a possible failure of the SWL this minimally invasive treatment should still be employed. 


\section{Acknowledgements}

Not applicable.

\section{Funding}

No funding was received.

\section{Availability of data and materials}

The datasets used and/or analyzed during the current study are available from the corresponding author on reasonable request.

\section{Authors' contributions}

$\mathrm{CP}$, VJ assesed the authenticity of raw data. CP, VJ, GDR, and DP conceived and designed the study. MI, AC, DP, NB, and CVT were involved with performing the surgery and other procedures. CP, VJ, DP, and GDR analyzed the data and drew conclusions. AC, CVT, and NB were involved in the drafting and writing of the manuscript. All authors read and approved the final manuscript.

\section{Ethics approval and consent to participate}

The study was approved by the 'C. I. Parhon' Clinical Hospital ethics committee with the approval no. 12/07.02.2020. Written informed consent was obtained from all the patients.

\section{Patient consent for publication}

Not applicable.

\section{Competing interests}

The authors state that they have no competing interests.

\section{References}

1. Scales CD Jr, Smith AC, Hanley JM and Saigal CS; Urologic Diseases in America Project: Prevalence of kidney stones in the United States. Eur Urol 62: 160-165, 2012.

2. Rassweiler JJ,Knoll T, Köhrmann KU, McAteer JA, Lingeman JE, Cleveland RO, Bailey MR and Chaussy C: Shock wave technology and application: An update. Eur Urol 59: 784-796, 2011.

3. Pearle MS: Shock-wave lithotripsy for renal calculi. N Engl J Med 367: 50-57, 2012.

4. Larcher P, De Cobelli O, Carmignani L, Franchini V, Meroni T, Panizzuti M, Strada G, Scardino E, Baccalin A and Rocco F: Prognostic parameters in extracorporeal lithotripsy. Arch Ital Urol Nefrol Androl 61: 361-365, 1989 (In Italian).

5. Türk C, Knoll T, Petrik A, Sarica K, Skolarikos A, Straub M and Seitz C: EAU guidelines on urolithiasis. European Association of Urology, Arnhem, 2018.

6. Preminger GM, Tiselius HG, Assimos DG, Alken P, Buck C, Gallucci M, Knoll T, Lingeman JE, Nakada SY, Pearle MS, et al: 2007 Guideline for the management of ureteral calculi. Eur Urol 52: 1610-1631, 2007

7. Nikoobakht MR, Emamzadeh A, Abedi AR, Moradi K and Mehrsai A: Transureteral lithotripsy versus extracorporeal shock wave lithotripsy in management of upper ureteral calculi: A comparative study. Urol J 4: 207-211, 2007.

8. Pearle MS, Lingeman JE, Leveillee R, Kuo R, Preminger GM, Nadler RB, Macaluso J, Monga M, Kumar U, Dushinski J, et al: Prospective, randomized trial comparing shock wave lithotripsy and ureteroscopy for lower pole caliceal calculi $1 \mathrm{~cm}$ or less. J Urol 173: 2005-2009, 2005.
9. Picozzi SC, Ricci C, Gaeta M, Casellato S, Stubinski R, Bozzini G, Pace G, Macchi A and Carmignani L: Urgent ureteroscopy as first-line treatment for ureteral stones: A meta-analysis of 681 patients. Urol Res 40: 581-586, 2012.

10. Irer B, Sahin MO, Erbatu O, Yildiz A, Ongun S, Cinar O, Cihan A, Sahin M, Sen V, Ucer O, et al: Impact of previous SWL on ureterorenoscopy outcomes and optimal timing for ureterorenoscopy after SWL failure in proximal ureteral stones. World J Urol 38: 769-774, 2020.

11. Kirkali Z, Esen AA, Hayran M, Gencbay A, Gidener S, Güven H and Güre A: The effect of extracorporeal electromagnetic shock waves on the morphology and contractility of rabbit ureter. J Urol 154: 1939-1943, 1995.

12. Park H, Park M and Park T: Two-year experience with ureteral stones: Extracorporeal shockwave lithotripsy v ureteroscopic manipulation. J Endourol 12: 501-504, 1998.

13. Abe T, Akakura K, Kawaguchi M, Ueda T, Ichikawa T, Ito H, Nozumi K and Suzuki K: Outcomes of shockwave lithotripsy for upper urinary-tract stones: A large-scale study at a single institution. J Endourol 19: 768-773, 2005.

14. Ur Rehman MF, Adnan M, Hassan A III, Humayun Akhtar F, Javed N and Ali F: Comparison of ureteroscopic pneumatic lithotripsy and extracorporeal shock wave lithotripsy for proximal ureteral calculi. Cureus 12: e7840, 2020.

15. Dell'Atti L and Papa S: Ten-year experience in the management of distal ureteral stones greater than $10 \mathrm{~mm}$ in size. G Chir 37: 27-30; 2016.

16. Soliman MG, Gameel T, El-Tatawy H and El-Abd AS: Extracorporeal shock wave lithotripsy for distal ureteric stones: Which is the ideal approach? Int Urol Nephrol 52: 2269-2274, 2020.

17. Holland R, Margel D, Livne PM, Lask DM and Lifshitz DA: Retrograde intrarenal surgery as second-line therapy yields a lower success rate. J Endourol 20: 556-559, 2006.

18. Kilinc MF, Doluoglu OG, Karakan T, Dalkilic A, Sonmez NC, Aydogmus Y and Resorlu B: Ureteroscopy in proximal ureteral stones after shock wave lithotripsy failure: Is it safe and efficient or dangerous? Can Urol Assoc J 9: E718-E722, 2015.

19. Philippou P, Payne D, Davenport K, Timoney AG and Keeley FX: Does previous failed ESWL have a negative impact of on the outcome of ureterorenoscopy? A matched pair analysis. Urolithiasis 41: 531-538, 2013.

20. Yanke B and Bagley D: Complications in ureteroscopy. In: Complications of Urologic Surgery and Practice. Loughlin KR (ed). Informa Healthcare, New York, NY, pp443-454, 2007.

21. Chaussy CG and Fuchs GJ: Current state and future developments of noninvasive treatment of human urinary stones with extracorporeal shock wave lithotripsy. J Urol 141: 782-789, 1989.

22. Tugcu V, Gürbüz G, Aras B, Gurkan L, Otunctemur A and Tasci AI: Primary ureteroscopy for distal-ureteral stones compared with ureteroscopy after failed extracorporeal lithotripsy. J Endourol 20: 1025-1029, 2006.

23. Akman T, Binbay M, Akcay M, Tekinarslan E, Kezer C, Ozgor F, Seyrek M and Muslumanoglu AY: Variables that influence operative time during percutaneous nephrolithotomy: An analysis of 1897 cases. J Endourol 25: 1269-1273, 2011.

24. Satava RM: Identification and reduction of surgical error using simulation. Minim Invasive Ther Allied Technol 14: 257-261, 2005.

25. Geavlete P, Georgescu D, Niţă G, Mirciulescu V and Cauni V: Complications of 2735 retrograde semirigid ureteroscopy procedures: A single-center experience. J Endourol 20: 179-185, 2005.

26. Ibrahim AK: Reporting ureteroscopy complications using the modified clavien classification system. Urol Ann 7: 53-57, 2015

27. Almusafer M and Jawad Al-Tawri AM: Complications of ureteroscopic stone lithotripsy: A multicenter local study. Hamdan Med J 12: 119-125, 2019.

28. Fuganti PE, Pires S, Branco R and Porto J: Predictive factors for intraoperative complications in semirigid ureteroscopy: Analysis of 1235 ballistic ureterolithotripsies. Urology 72: 770-774, 2008.

29. Ogreden E, Demirelli E, Aksu M, Tok DS and Oğuz U: Early ureteroscopic lithotripsy in acute renal colic caused by ureteral calculi. Int Urol Nephrol 52: 15-19, 2020.

30. Rosenthal R, Hoffmann H, Clavien PA, Bucher HC and Dell-Kuster S: Definition and classification of intraoperative complications (CLASSIC): Delphi study and pilot evaluation. World J Surg 39: 1663-1671, 2015. 
31. Traxer $\mathrm{O}$ and Thomas A: Prospective evaluation and classification of ureteral wall injuries resulting from insertion of a ureteral access sheath during retrograde intrarenal surgery. J Urol 189: 580-584, 2013.

32. Tepeler A, Resorlu B, Sahin T, Sarikaya S, Bayindir M, Oguz U, Armagan A and Unsal A: Categorization of intraoperative ureteroscopy complications using modified Satava classification system. World J Urol 32: 131-136, 2014.

33. Pricop C, Maier A, Negru D, Malau O, Orsolya M, Radavoi D and Serban DR: Extracorporeal shock waves lithotripsy versus retrograde ureteroscopy: Is radiation exposure a criterion when we choose which modern treatment to apply for ureteric stones? Bosn J Basic Med Sci 14: 254-258, 2014.
34. Abid N, Ravier E, Promeyrat X, Codas R, Fehri HF, Crouzet S and Martin X: Decreased radiation exposure and increased efficacy in extracorporeal lithotripsy using a new ultrasound stone locking system. J Endourol 29: 1263-1269, 2015.

35. Kapogiannis F, Spartalis E, Fasoulakis K, Tsourouflis G, Dimitroulis D and Nikiteas NI: Laparoscopic and robotic management of ureteral stricture in adults. In Vivo 34: 965-972, 2020.

36. Xiong M, Zhu X, Chen D, Hossain MA, Xie Y, Gou X and Deng Y: Post ureteroscopic stone surgery ureteral strictures management: A retrospective study. Int Urol Nephrol 52: 841-849, 2020 